\title{
ON COMPLETE INDEPENDENCE OF CERTAIN SETS OF POSTULATES FOR FIELDS*
}

\author{
BY N. H. MCCOY
}

1. Introduction. Definitions of a field by sets of independent postulates have been given by a number of authors, $\dagger$ but in most cases the question of complete independence in the sense of E. H. Moore $\ddagger$ has not been considered. The primary purpose of this note is to present a method which may be used to advantage in the study of complete independence of any set of field postulates except those which contain a postulate specifying the cardinal number of the system as a given finite number. The method will be presented by applying it to establish the complete independence of a set of seven postulates, essentially the Definition 1 of Huntington. $\S$ In $\S 4$ a very short proof of the complete independence of Hurwitz's\| five postulates for fields is also given. This set was previously shown by Bernstein $\uparrow$ to be completely independent.

2. The Seven Postulates. Let $K$ be a set of undefined elements $a, b, c, \cdots$. We assume the existence of two uniquely defined binary operations $\oplus$ and $\odot$ on the elements of $K$ as well as an equality $\ominus$ which is reflexive, symmetric, and transitive.** The system $[K, \oplus, \odot, \ominus]$ will be a field if it has the following seven properties $\nmid \dagger \dagger$

* Presented to the Society, December 29, 1932.

$\uparrow$ For a list of references see a recent article by W. A. Hurwitz, Annals of Mathematics, (2), vol. 33 (1932), pp. 403-405. See also an abstract by C. R. Worth, this Bulletin, vol. 38 (1932), p. 350 .

$\ddagger$ E. H. Moore, Introduction to a Form of General Analysis, New Haven Mathematical Colloquium, Yale University Press, p. 82.

$\S$ E. V. Huntington, Transactions of this Society, vol. 4 (1903), pp. 31-37.

II W. A. Hurwitz, Annals of Mathematics, (2), vol. 15 (1913), pp. 93-100.

ๆ B. A. Bernstein, Annals of Mathematics, (2), vol. 23 (1921-22), pp. 313 316.

** See, for example, O. Haupt, Einführung in die Algebra, vol. 1, p. 2. For non-equality, we shall say "does not $\Theta$."

$\dagger \dagger$ E. V. Huntington, loc. cit., p. 34. This set of properties is the Definition 1 of Huntington with the exception that $P 6$ is made slightly stronger in statement. This set was later modified by Huntington; see Transactions of this Society, vol. 6 (1905), pp. 181-193. 
P1. If $a, b, c, a \oplus b, b \oplus c$, and $a \oplus b(\oplus c)$ belong to $K$, then $(a \oplus b) \oplus c \ominus a \oplus(b \oplus c)$.

P2. If $a, b$, and $b \oplus a$ belong to $K$, then $a \oplus b \ominus b \oplus a$.

$P 3$. If $a$ and $b$ belong to $K$, there is an element $x$ of $K$ such that $a \oplus x \ominus b$.

P4. If $a, b, c, a \odot b, b \odot c$, and $a \odot(b \odot c)$ belong to $K$, then $(a \odot b) \odot c \ominus a \odot(b \odot c)$.

$P 5$. If $a, b, b \odot a$ belong to $K$, then $a \odot b \ominus b \odot a$.

$P 6$. If $a$ and $b$ belong to $K$ and $a \oplus a$ does not $\ominus a$, there is an element $y$ of $K$ such that $a \odot y \ominus b$.

P7. If $a, b, c, b \oplus c, a \odot b, a \odot c$, and $(a \odot b) \oplus(a \odot c)$ belong to $K$, then $a \odot(b \oplus c) \ominus(a \odot b) \oplus(a \odot c)$.

We shall now show that this set of properties is completely independent.

3. Proof of Complete Independence. To establish the complete independence of the above seven properties we need to show the existence of $2^{7}$ systems having each of the possible characters with respect to these properties. Each of the systems used below is an infinite system and each one is actually closed with respect to the operations $\oplus$ and $\odot$.

Let $S_{i}$ denote a system $\left[K_{i},(+)_{i},(\cdot)_{i},(=)_{i}\right]$, and let $a_{i}, b_{i}, \cdots$ denote elements of $K_{i},(i=1,2, \cdots)$. We now define the multiple system $\left(S_{i}, S_{j}, \cdots, S_{k}\right)$, formed from the component systems $S_{i}, S_{j}, \cdots, S_{k}$, and consisting of all elements of the form $\left(a_{i}, a_{j}, \cdots, a_{k}\right)$. The symbols $\oplus, \odot, \ominus$ for this multiple system will be denoted by $[+],[\cdot]$ and $[=]$ respectively, and are defined as follows:

$$
\left(a_{i}, a_{j}, \cdots, a_{k}\right)[+]\left(b_{i}, b_{j}, \cdots, b_{k}\right)
$$

is the element

$$
\begin{aligned}
& \left(a_{i}(+)_{i} b_{i}, a_{j}(+)_{j} b_{j}, \cdots, a_{k}(+)_{k} b_{k}\right) \\
& \left(a_{i}, a_{j}, \cdots, a_{k}\right)[\cdot]\left(b_{i}, b_{j}, \cdots, b_{k}\right)
\end{aligned}
$$

is the element

$$
\begin{aligned}
& \left(a_{i}(\cdot)_{i} b_{i}, a_{j}(\cdot)_{j} b_{j}, \cdots, a_{k}(\cdot)_{k} b_{k}\right) \\
& \left(a_{i}, a_{j}, \cdots, a_{k}\right)[=]\left(b_{i}, b_{j}, \cdots, b_{k}\right)
\end{aligned}
$$

means

$$
a_{i}(=)_{i} b_{i}, a_{j}(=)_{j} b_{j}, \cdots, a_{k}(=)_{k} b_{k} .
$$


The following theorems are now evident.

THEOREM 1. If any one of the component systems $S_{i}, S_{j}, \cdots, S_{k}$ does not have property $\operatorname{Pr}$, the multiple system $\left(S_{i}, S_{j}, \cdots, S_{k}\right)$ does not have property $\operatorname{Pr},(r=1,2, \cdots, 7)$.

Theorem 2. If each of the component systems $S_{i}, S_{j}, \cdots, S_{k}$ has property $\operatorname{Pr},(r \neq 6)$, the multiple system $\left(S_{i}, S_{j}, \cdots, S_{k}\right)$ also has this property.

In order to state the corresponding theorem for $P 6$, we make use of the following two auxiliary properties:

I. If $a$ belongs to $K$, then $a \oplus a \ominus a$.

II. If $a$ and $b$ belong to $K$, there exists an element $y$ of $K$ such that $a \odot y \ominus b$.

We now prove the following theorem.

THEOREM 3. If each of the component systems $S_{i}, S_{j}, \cdots, S_{k}$ has property $P 6$, the system $\left(S_{i}, S_{j}, \cdots, S_{k}\right)$ also has this property if (1) each of the component systems has property I, or (2) each of the component systems has property II, or (3) some one of the component systems has neither of the properties I and II and the remaining ones have both these properties.

It is readily verified that the multiple system $\left(S_{i}, S_{j}, \cdots, S_{k}\right)$ has property $P 6$ in cases (1) and (2). In case (3) suppose that $S_{i}$ has neither property I nor property II. If

$$
\left(a_{i}, a_{j}, \cdots, a_{k}\right)[\cdot]\left(y_{i}, y_{\jmath}, \cdots, y_{k}\right)[=]\left(b_{i}, b_{j}, \cdots, b_{k}\right)
$$

admits no solution for $\left(y_{i}, y_{j}, \cdots, y_{k}\right)$, then the equation $a_{i}(\cdot)_{i} y_{i}(=)_{i} b_{i}$ admits no solution in $K_{i}$, and since $S_{i}$ has property $P 6$, we must have $a_{i}(+)_{i} a_{i}(=)_{i} a_{i}$. Hence

$$
\left(a_{i}, a_{j}, \cdots, a_{k}\right)[+]\left(a_{i}, a_{j}, \cdots, a_{k}\right)[=]\left(a_{i}, a_{j}, \cdots, a_{k}\right),
$$

and the multiple system thus has property $P 6$.

The table defines ten systems which are fundamental in our proof of the complete independence of the seven properties. Henceforth, as in the table, $S_{i}$ will denote a system which has each of the seven properties with the exception of $P i, S_{i j}$ a system which has neither of the properties $P i$ and $P j$ but each of the other properties. Thus systems $S_{1}, S_{2}, \cdots, S_{7}$ show the 
ordinary independence of the set.* The last column of the table gives the character of each system with respect to properties I and II, and is for convenience in applying Theorem 3.

By means of Theorems 1 and 2 we can construct at once from $S_{1}, S_{2}, \cdots, S_{7}$ a system not having property $P 6$ but having any given combination of the remaining properties. For if we wish, for example, a system having each of the properties with the exception of $P 1, P 5$, and $P 6$, we have merely to use the system $\left(S_{1}, S_{5}, S_{6}\right)$. This disposes of the 64 systems which do not have property $P 6$ and henceforth we shall only consider systems which have this property.

Table of Fundamental Systems

\begin{tabular}{|c|c|c|c|c|c|c|}
\hline System & $K$ & $a \oplus b$ & $a \odot b$ & $a \ominus b$ & I & II \\
\hline$S_{1}$ & Positive reals & $+(a b)^{1 / 2}$ & $a b$ & $a=b$ & + & + \\
\hline$S_{2}$ & Positive rationals & $b$ & $a b$ & $a=b$ & + & + \\
\hline$S_{3}$ & Positive rationals & $a+b$ & $a b$ & $a=b$ & - & + \\
\hline \multirow[t]{2}{*}{$S_{4}$} & $\begin{array}{l}\text { Number pairs } \\
a=\left(a_{1}, a_{2}\right) \text {, with } a_{1} \text {, }\end{array}$ & & & & & \\
\hline & $a_{2}$ rational & $\left(a_{1}+b_{1}, a_{2}+b_{2}\right)$ & $\left(\begin{array}{l}\left(a_{1} b_{1}-a_{2} b_{2}\right. \\
\left.-a_{1} b_{2}-a_{2} b_{1}\right)\end{array}\right.$ & $\left\{\begin{array}{l}a_{1}=b_{1} \\
a_{2}=b_{2}\end{array}\right.$ & - & - \\
\hline$S_{5}$ & Rationals & $a+b$ & $b$ & $a=b$ & - & + \\
\hline$S_{6}$ & Rationals & $a+b$ & 0 & $a=b$ & - & - \\
\hline$S_{7}$ & Rationals & $a+b$ & $a+b$ & $a=b$ & - & + \\
\hline$S_{34}$ & Same as in $S_{4}$ & $(0,0)$ & Same as in $S_{4}$ & $\begin{array}{c}\text { Same as } \\
\text { in } S_{4}\end{array}$ & - & - \\
\hline$S_{45}$ & Rationals & $a+b$ & $-b$ & $a=b$ & - & + \\
\hline$S_{47}$ & Rationals & $a+b$ & $-(a+b)$ & $a=b$ & - & + \\
\hline
\end{tabular}

Now each of the systems $S_{1}, S_{2}, S_{3}, S_{5}, S_{7}$ has property II, and by Theorem 3 , property $P 6$ will be present in any multiple system constructed from them. We can therefore, as above, construct a system which has properties $P 6$ and $P 4$ and any combination of the remaining properties. Hence in order to establish complete independence we only need to exhibit 32 systems having property $P 6$ and not having property $P 4$ but admitting each possible character with respect to the remaining five properties.

* The systems $S_{1}, S_{2}, S_{3}, S_{4}$ were used by Huntington (loc. cit.), the last of these being due to Dickson. Systems $S_{5}, S_{6}, S_{7}$ were used by Bernstein (loc. cit.). 
If $S$ denotes any one of these 32 remaining systems and if it has property $P 1$, then the system $\left(S_{1}, S\right)$ will be a system having the same character as $S$ with the exception that property $P 1$ is absent. This is true by Theorem 3 as $S_{1}$ has both properties I and II. A similar statement holds for property $P 2$. Thus the existence of the remaining 32 systems will have been established when we exhibit the eight systems having properties $P 1, P 2$, and $P 6$ and not having property $P 4$ but having each combination of the other properties. It may be verified that the following eight systems satisfy these requirements: $S_{4}, S_{34}, S_{45}, S_{47},\left(S_{3}, S_{45}\right)$, $\left(S_{3}, S_{47}\right),\left(S_{45}, S_{7}\right)$, and $\left(S_{3}, S_{5}, S_{47}\right)$. This completes the proof of the complete independence of the seven properties.

4. Complete Independence of Hurwitz's Five Postulates. Hurwit $z^{*}$ has given a definition of a field by means of a set of five postulates, which are our $P 3, P 6, P 7$, and the following two:

$P 12$. If $a, b, c, a \oplus b, c \oplus b$, and $a \oplus(c \oplus b)$ belong to $K$, then $(a \oplus b) \oplus c \ominus a \oplus(c \oplus b)$.

P45. If $a, b, c, a \odot b, c \odot b$, and $a \odot(c \odot b)$ belong to $K$, then $(a \odot b) \odot c \ominus a \odot(c \odot b)$.

It is seen that Theorems 1, 2, and 3 hold also for this set of five properties. Ordinary independence is shown by the systems $S_{3}, S_{6}, S_{7}, S_{1}$, and $S_{5}$. But with the exception of $S_{6}$ each of these systems has property II. Hence it is easily verified that any multiple system formed from these will have no one of the properties which is lacking in any of the component systems and will have each property which is present in each component system. From the above five systems we can therefore form at once a multiple system having any desired combination of the five properties. Thus the set is completely independent. Another proof of this fact has been given by Bernstein. $\dagger$

Smith College

* W. A. Hurwitz, Annals of Mathematics, (2), vol. 15 (1913), pp. 93-100.

$\dagger$ Loc. cit. See also this Bulletin, vol. 28 (1922), pp. 397-399. 